\title{
PRECONDITIONED LANCZOS METHODS FOR THE MINIMUM EIGENVALUE OF A SYMMETRIC POSITIVE DEFINITE TOEPLITZ MATRIX*
}

\author{
MICHAEL K. NG ${ }^{\dagger}$
}

\begin{abstract}
In this paper, we apply the preconditioned Lanczos (PL) method to compute the minimum eigenvalue of a symmetric positive definite Toeplitz matrix. The sine transform-based preconditioner is used to speed up the convergence rate of the PL method. The resulting method involves only Toeplitz and sine transform matrix-vector multiplications and hence can be computed efficiently by fast transform algorithms. We show that if the symmetric Toeplitz matrix is generated by a positive $2 \pi$-periodic even continuous function, then the PL method will converge sufficiently fast. Numerical results including Toeplitz and non-Toeplitz matrices are reported to illustrate the effectiveness of the method.
\end{abstract}

Key words. Toeplitz matrix, sine transform matrix, fast sine transform, preconditioning, Lanczos method

AMS subject classifications. 65F10, 65Y20

PII. S1064827597330169

1. Introduction. An $n$-by- $n$ matrix $T_{n}$ is said to be Toeplitz if

$$
T_{n}=\left[\begin{array}{ccccc}
t_{0} & t_{-1} & \cdots & t_{2-n} & t_{1-n} \\
t_{1} & t_{0} & t_{-1} & & t_{2-n} \\
\vdots & t_{1} & t_{0} & \ddots & \vdots \\
t_{n-2} & & \ddots & \ddots & t_{-1} \\
t_{n-1} & t_{n-2} & \cdots & t_{1} & t_{0}
\end{array}\right]
$$

i.e., $T_{n}$ is constant along its diagonals. There are many efficient direct methods that exploit Toeplitz structure to invert Toeplitz matrices or to solve Toeplitz systems $T_{n} x=b[15,13]$. There are also preconditioned conjugate gradient methods for solving Toeplitz systems with $O(n \log n)$ operations [4].

Recent progress in signal processing and estimation has generated considerable interest in the problem of computing the minimum eigenvalue of a Toeplitz matrix. Numerical solution of the Toeplitz eigenvalue problem has recently received attention $[6,10,22,23,16]$. In particular, Cybenko and Van Loan [6] presented a method for using Levinson's algorithm [15] to find the minimum eigenvalue of an $n$-by- $n$ Hermitian Toeplitz matrix with $O\left(n^{2}\right)$ operations. In [22, 23], Trench extended their method and gave an iterative method for computing arbitrary eigenvalues and associated eigenvectors of Hermitian Toeplitz and Toeplitz-plus-Hankel matrices at a cost of $O\left(n^{2}\right)$ per eigenvalue. On the other hand, there is an extensive literature on the asymptotic distribution of the eigenvalues of a sequence of Toeplitz matrices. In this case, the diagonals of the Toeplitz matrix are given by the Fourier coefficients of the periodic function $f$, see [9].

*Received by the editors June 1, 1997; accepted for publication (in revised form) April 6, 1999; published electronically May 17, 2000.

http://www.siam.org/journals/sisc/21-6/33016.html

${ }^{\dagger}$ Department of Mathematics, The University of Hong Kong, Pokfulam Road, Hong Kong (mng@maths.hku.hk). The research was supported in part by HKU CRCG grants 10201824 and 10201939. 


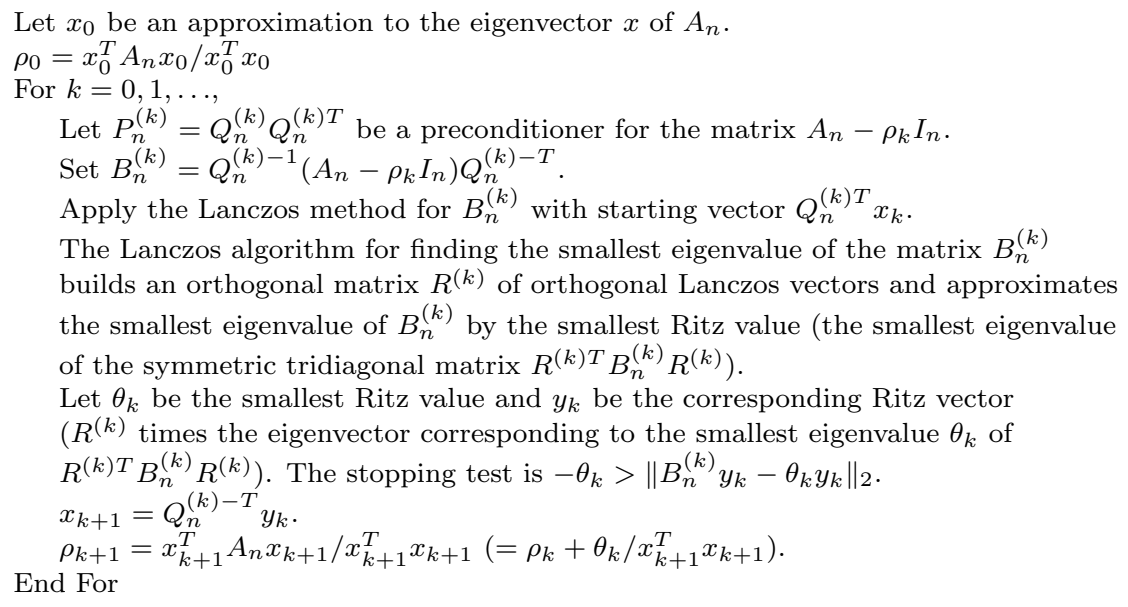

The purpose of this paper is to use the preconditioned Lanczos (PL) method for computing the minimum eigenvalue of a symmetric positive definite Toeplitz matrix. The PL method is stated as in Table 1.1. In Table 1.1, the outside loop of the method updates a certain preconditioned matrix and the inside loop applies the Lanczos method to the preconditioned matrix $B_{n}^{(k)}$. We compute the smallest Ritz value and the corresponding Ritz vector of $B_{n}^{(k)}$ and then transform it back to get $x_{k+1}$, an approximate eigenvector of $A_{n}$. The Rayleigh quotient $\rho_{k+1}$ of $x_{k+1}$ is an approximate eigenvalue of $A_{n}$. Morgan and Scott [17] showed both theoretically and numerically that the PL method is effective. In particular, they proved that the iterate in the PL algorithm converges to the minimum eigenvalue of $A_{n}$ at an asymptotically quadratic rate.

The motivation behind using the preconditioned Lanczos method in this paper is that the Toeplitz matrix-vector product $T_{n} v$ can be computed efficiently. For instance, the matrix-vector product can be computed by the fast Fourier transform (FFT) by first embedding $T_{n}$ into a $2 n$-by- $2 n$ circulant matrix. The FFT for a real $n$-vector based on the split-radix method requires $\left[4 n \nu / 3-17 n / 9-(-1)^{\nu} / 9+3\right]$ additions and $\left[2 n \nu / 3-19 n / 9-(-1)^{\nu} / 9+3\right]$ multiplications, where $n=2^{\nu}$ for positive integers $\nu$ (see [1]). Thus when $n=2^{\nu}$, the cost of the matrix-vector product requires $O(n \log n)$ operations (see [4]). In order to compete with other methods [6,22], the preconditioner should be chosen so that the Lanczos method, when applied to the preconditioned matrix, converges sufficiently fast in the inner loop. It is well known that the Lanczos method converges very quickly if the desired eigenvalues are well separated from the rest of the spectrum [19,21].

In this paper, we employ the sine transform-based preconditioner to precondition the Toeplitz matrix in the Lanczos algorithm. The idea of using preconditioned conjugate gradient methods with sine transform-based preconditioners for solving Toeplitz systems has been studied recently by various researchers, see $[2,3,5,11]$. In these papers, the symmetric Toeplitz matrix $T_{n}$ is assumed to be generated by a $2 \pi$-periodic even function. It has been shown that if the function is positive and 
continuous, then the conjugate gradient method when applied to solve these sine transform preconditioned systems, will converge superlinearly, for instance (see [5]).

The outline of this paper is as follows. In section 2, we recall some of the results in approximating a given matrix by matrices that can be diagonalized by the discrete sine transform matrix. In section 3 , we use the optimal sine transform approximation to construct preconditioners for the iteration matrices in the PL method. We will show that if the symmetric Toeplitz matrix is generated by a positive $2 \pi$-periodic even continuous function, then the spectra of the preconditioned matrices in the PL algorithm will be clustered around 1 . We prove that if the spectra of the preconditioned matrices are clustered around 1, the Lanczos method, when applied to find the minimum eigenvalue of the preconditioned matrix, will converge sufficiently fast. In section 4, we discuss an application to signal processing and extend our method to compute the minimum eigenvalue of a non-Toeplitz matrix. Finally, we describe the results of numerical experiments with the algorithm in section 5 .

2. Sine transform approximations. In the following, we assume that the entries $t_{j}$ in (1.1) are given by

$$
t_{j}=\frac{1}{\pi} \int_{0}^{\pi} f(\theta) \cos j \theta d \theta, \quad j=0, \pm 1, \pm 2, \ldots .
$$

The function $f$ is called the generating function of the Toeplitz matrix $T_{n}$. We emphasize that this class of Toeplitz matrices arises in some practical applications; see $[4,9]$ and the references therein. For this class of Toeplitz matrices, we note that there is a close relationship between the spectrum of $T_{n}$ and its generating function $f$.

Theorem 2.1 (Grenander and Szegö [9]). Let $f$ be a continuous function on $[0,2 \pi]$. Then the spectrum $\lambda\left(T_{n}\right)$ of $T_{n}$ satisfies

$$
\lambda\left(T_{n}\right) \subseteq\left[f_{\min }, f_{\max }\right] \quad \forall n \geq 1,
$$

where $f_{\min }$ and $f_{\max }$ are the minimum and maximum values of $f$, respectively. Moreover, the eigenvalues $\lambda_{j}\left(T_{n}\right), j=0,1, \ldots, n-1$, are equally distributed as $f(2 \pi j / n)$, i.e.,

$$
\lim _{n \rightarrow \infty} \frac{1}{n} \sum_{j=0}^{n-1}\left[g\left(\lambda_{j}\left(T_{n}\right)\right)-g\left(f\left(\frac{2 \pi j}{n}\right)\right)\right]=0
$$

for any continuous function $g$ defined on $[0,2 \pi]$.

The equal distribution of eigenvalues of Toeplitz matrices indicates that the eigenvalues will not be clustered in general [9, p. 65]. Therefore, when the Lanczos method is applied to compute the minimum eigenvalue of this Toeplitz matrix, it does not converge very quickly; see the numerical results in section 5 . To speed up the convergence of the Lanczos method, we construct sine transform based-preconditioners for this class of Toeplitz matrices.

The $(j, k)$ entry of the $n$-by- $n$ discrete sine transform matrix $\Psi_{n}$ is given by

$$
\sqrt{\frac{2}{n+1}} \sin \left(\frac{\pi j k}{n+1}\right), \quad 1 \leq j, k \leq n .
$$

We note that $\Psi_{n}$ are symmetric and orthogonal, i.e., $\Psi_{n}=\Psi_{n}^{T}$ and $\Psi_{n} \Psi_{n}^{T}=I_{n}$. For any $n$-vector $v$, the matrix-vector product $\Psi_{n} v$ can be done in $O(n \log n)$ real operations by the fast sine transforms (FSTs); see, for instance, Yip and Rao [25]. 
Let $\mathbf{S}$ be the vector space over the field of real numbers containing all $n$-by- $n$ matrices that can be diagonalized by the discrete sine transform matrix $\Psi_{n}$, i.e.,

$$
\mathbf{S}=\left\{\Psi_{n} \Lambda_{n} \Psi_{n} \mid \Lambda_{n} \text { is diagonal }\right\} .
$$

The authors of $[2,3,5,11]$ proposed the use of matrices that can be diagonalized by the sine transform matrix $\Psi_{n}$ to precondition Toeplitz matrices in conjugate gradient iterations. The motivation is to exploit the fast inversion of these sine transform-based matrices. They showed that the conjugate gradient iteration converges superlinearly for a wide range of Toeplitz matrices; see [4].

Given an $n$-by- $n$ matrix $A_{n}$, we are interested in finding a matrix $S_{n} \in \mathbf{S}$ which minimizes $\left\|S_{n}-A_{n}\right\|_{F}$ in the Frobenius norm $\|\cdot\|_{F}$. We will denote the minimizer by $P_{n}$ and call it the optimal sine transform approximation to $A_{n}$. The following lemma gives some basic properties of $P_{n}$.

Lemma 2.2 (Chan, Ng, and Wong [5]). Let $A_{n}$ be an n-by-n symmetric matrix and $P_{n}$ be the minimizer of $\left\|S_{n}-A_{n}\right\|_{F}$ over all $S_{n} \in \mathbf{S}$. Then $P_{n}$ is uniquely determined by $A_{n}$ and is given by

$$
P_{n}=\Psi_{n} \Delta_{n} \Psi_{n}
$$

where $\Delta_{n}$ denotes the diagonal matrix whose diagonal is equal to the diagonal of the matrix $\Psi_{n} A_{n} \Psi_{n}$, i.e.,

$$
\left[\Delta_{n}\right]_{j, j}=\left[\Psi_{n} A_{n} \Psi_{n}\right]_{j, j}
$$

Furthermore,

$$
\lambda_{\min }\left(A_{n}\right) \leq \lambda_{\min }\left(\Delta_{n}\right) \leq \lambda_{\max }\left(\Delta_{n}\right) \leq \lambda_{\max }\left(A_{n}\right) .
$$

In particular, if $A_{n}$ is positive definite, then $P_{n}$ is also positive definite.

We remark that this spectral property will be used to generate the starting value $\rho_{0}^{(n)}$ and the starting vector $x_{0}$ (cf. Table 1.1) in the PL algorithm.

We note that forming $P_{n}$ by computing all the diagonal entries of $\Psi_{n} A_{n} \Psi_{n}$ as in (2.1) requires $O\left(n^{2} \log n\right)$ operations. Chan, $\mathrm{Ng}$, and Wong [5] gave another approach for constructing $P_{n}$ which reduces the cost to $O\left(n^{2}\right)$ operations. Before we describe how the matrix $P_{n}$ is formed, we let $U_{i}, i=1, \ldots, n$, be $n$-by- $n$ matrices with the $(j, k)$ th entry given by

$$
\left[U_{i}\right]_{j, k}= \begin{cases}1 & \text { if }|j-k|=i-1 \\ -1 & \text { if } j+k=i-2 \\ -1 & \text { if } j+k=2 n-i+3 \\ 0 & \text { otherwise }\end{cases}
$$

We note that each $U_{i}$ is a sparse matrix with at most $2 n$ nonzero entries. Also, we let

$$
r=\left(1_{n}^{T}\left(U_{1} \circ A_{n}\right) 1_{n}, 1_{n}^{T}\left(U_{2} \circ A_{n}\right) 1_{n}, \ldots, 1_{n}^{T}\left(U_{n} \circ A_{n}\right) 1_{n}\right)^{T},
$$

where $1_{n}=[1,1, \ldots, 1]^{T}$ and $\circ$ is the Hadamard product. Now we can give explicit formulae for the entries of the minimizer $P_{n}$.

Lemma 2.3. (Chan, Ng, and Wong [5]) Let $A_{n}=\left[a_{j k}\right]$ be an n-by-n symmetric matrix and $P_{n}$ be the minimizer of $\left\|S_{n}-A_{n}\right\|_{F}$ over all $S_{n} \in \mathbf{S}$. Denote the first 
column of $P_{n}$ by $p$. If $s_{o}$ and $s_{e}$ are defined, respectively, to be the sum of the odd and even index entries of $r$ where the vector $r$ is given in (2.4), then we have

$$
[p]_{1}=\frac{1}{2(n+1)}\left(2[r]_{1}-[r]_{3}\right), \quad[p]_{i}=\frac{1}{2(n+1)}\left([r]_{i}-[r]_{i+2}\right), \quad i=2, \ldots, n-2,
$$

with

$$
[p]_{n-1}=\frac{1}{2(n+1)}\left(s_{o}+[r]_{n-1}\right), \quad[p]_{n}=\frac{1}{2(n+1)}\left(2 s_{e}+[r]_{n}\right)
$$

if $n$ is even and

$$
[p]_{n-1}=\frac{1}{2(n+1)}\left(s_{e}+[r]_{n-1}\right), \quad[p]_{n}=\frac{1}{2(n+1)}\left(2 s_{o}+[r]_{n}\right)
$$

if $n$ is odd.

We remark that if $A_{n}$ has no special structure, then the vector $r$ can be computed in $O\left(n^{2}\right)$ operations because $U_{i}$ are sparse with only $O(n)$ nonzero entries each. Therefore $P_{n}$ can be computed in $O\left(n^{2}\right)$ operations. However, if $A_{n}$ is a Toeplitz matrix, then the cost can be reduced to $O(n)$ operations. In particular, if $\left[t_{0}, t_{1}, \ldots, t_{n-1}\right]^{T}$ is the first column of an $n$-by- $n$ symmetric Toeplitz matrix, then the first column of the corresponding optimal sine transform-based preconditioner $P_{n}$ is given by

$$
p_{k}= \begin{cases}t_{0}-\left(\frac{n-2}{n+1}\right) t_{2}, & k=1, \\ t_{1}-\left(\frac{n-3}{n+1}\right) t_{3}, & k=2, \\ \left(\frac{n-k+3}{n+1}\right) t_{k-1}-\left(\frac{n-k-1}{n+1}\right) t_{k+1}, & k=3,4, \ldots, n-2, \\ \left(\frac{4}{n+1}\right) t_{n-2}, & k=n-1, \\ \left(\frac{3}{n+1}\right) t_{n-1}, & k=n .\end{cases}
$$

In the next section, we will use the optimal sine transform approximation to construct preconditioners for the iteration matrices in the PL method.

3. The convergence of preconditioned Lanczos methods. In this section, we discuss the convergence of the preconditioned Lanczos method for Toeplitz matrices. We first note the following lemma.

LEMma 3.1. Let $\left\{A_{n}\right\}$ be a sequence of n-by-n symmetric positive definite matrices and let $\Delta_{n}$ denote the diagonal matrix whose diagonal is equal to the diagonal of the matrix $\Psi_{n} A_{n} \Psi_{n}$. If

$$
\lambda_{\min }\left(A_{n}\right) \leq \rho_{0}^{(n)}<\alpha_{n} \equiv \min _{1 \leq j \leq n}\left\{\left[\Delta_{n}\right]_{j, j}\right\}
$$

and the preconditioners $P_{n}^{(k)}$ are constructed by

$$
P_{n}^{(k)}=\Psi_{n} \Delta_{n} \Psi_{n}-\rho_{k}^{(n)} I_{n}, \quad k \geq 0,
$$


where $\rho_{0}^{(n)}$ is the starting value and $\rho_{k}^{(n)}$ is generated in the $P L$ algorithm, then the matrices $P_{n}^{(k)}$ are positive definite for $k \geq 0$. Moreover, if $\inf _{n}\left|\alpha_{n}-\rho_{0}^{(n)}\right| \geq \delta>0$, where $\delta$ is a constant independent of $n$, then $\left\|P_{n}^{(k)-1}\right\|_{2}$ is uniformly bounded for all $n$ and $k$.

Proof. Using (2.3), it is easy to show that the matrix $\Delta_{n}$ is positive-definite. Therefore, the matrix $P_{n}^{(0)}$ is also positive definite. According to the PL algorithm (cf. Table 1.1), we have

$$
\rho_{k+1}^{(n)}=\rho_{k}^{(n)}+\frac{\theta_{k}}{x_{k+1}^{T} x_{k+1}}
$$

and $\theta_{k}$ is nonpositive [17, Theorem 1], therefore the sequence $\left\{\rho_{k}^{(n)}\right\}_{k=0}^{\infty}$ is a nonincreasing sequence. It follows that $P_{n}^{(k)}$ is positive definite for $k \geq 0$. Hence if $\inf _{n}\left|\alpha_{n}-\rho_{0}^{(n)}\right| \geq \delta>0$, we have

$$
\left\|P_{n}^{(k)-1}\right\|_{2} \leq \frac{1}{\delta} \quad \forall n \geq 1, k \geq 0 .
$$

The result follows.

We note from Table 1.1 that the Cholesky factorization of the preconditioner $P_{n}^{(k)}$ is required, i.e.,

$$
P_{n}^{(k)}=Q_{n}^{(k)} Q_{n}^{(k) T}
$$

In our case, since $P_{n}^{(k)}$ can be diagonalized by the discrete sine transform matrix $\Psi_{n}$, the matrix $Q_{n}^{(k)}$ is given by

$$
Q_{n}^{(k)}=\Psi_{n}\left(\Delta_{n}-\rho_{k}^{(n)} I_{n}\right)^{1 / 2}
$$

with no extra computations using the spectral decomposition of the preconditioner. Next we show that the spectra of the preconditioned Toeplitz matrices $Q_{n}^{(k)-1}\left(T_{n}-\right.$ $\left.\rho_{k}^{(n)} I_{n}\right) Q_{n}^{(k)-T}$ are clustered around 1 in the Lanczos iteration.

THEOREM 3.2. Let $f$ be a positive continuous function on $[0, \pi]$ and $\left\{T_{n}\right\}$ be a sequence of Toeplitz matrices generated by $f$. If $\inf _{n}\left|\alpha_{n}-\rho_{0}^{(n)}\right| \geq \delta>0$, where $\delta$ is a constant independent of $n$, then for any given $\epsilon>0$, there exist positive integers $N_{1}$ and $N_{2}$ such that for all $n>N_{1}$ and $k \geq 0$, at most $N_{2}$ eigenvalues of the matrices $Q_{n}^{(k)-1}\left(T_{n}-\rho_{k}^{(n)} I_{n}\right) Q_{n}^{(k)-T}$ lie outside the interval $(1-\epsilon, 1+\epsilon)$. matrix

Proof. Using the matrix decomposition $P_{n}^{(k)-1}=Q_{n}^{(k)-T} Q_{n}^{(k)-1}$, we note that the

$$
Q_{n}^{(k)-1}\left(T_{n}-\rho_{k}^{(n)} I_{n}\right) Q_{n}^{(k)-T}
$$

is similar to the matrix

$$
I_{n}+P_{n}^{(k)-1}\left(T_{n}-\rho_{k}^{(n)} I_{n}-P_{n}^{(k)}\right)
$$

It has been proved in [5] that for any given $\epsilon>0$, there exist positive integers $N_{1}$ and $N_{2}$ such that for all $n>N_{1}$, at most $N_{2}$ eigenvalues of the matrices $T_{n}-$ $\Psi_{n} \Delta_{n} \Psi_{n}\left(=T_{n}-P_{n}^{(0)}\right)$ have absolute values exceeding $\epsilon$. By noting (3.1) and the fact that $\left\|P_{n}^{(k)-1}\right\|_{2}$ is uniformly bounded, the spectra of $Q_{n}^{(k)-1}\left(T_{n}-\rho_{k}^{(n)} I_{n}\right) Q_{n}^{(k)-T}$ are clustered around 1 . 
The following theorem [8, Corollary 9.1.4] states the convergence rate of the estimate of the smallest eigenvalue in the Lanczos method for a symmetric matrix in the exact arithmetic.

THEOREM 3.3. Let $A_{n}$ be an $n$-by-n symmetric matrix with eigenvalues $\lambda_{1}^{(n)} \geq$ $\cdots \geq \lambda_{n}^{(n)}$ and corresponding orthonormal eigenvectors $z_{1}^{(n)}, \ldots, z_{n}^{(n)}$. If $\theta_{1}^{(n)} \geq \cdots \geq$ $\theta_{j}^{(n)}$ are the eigenvalues of the matrix $W_{j}$ obtained after $j$ steps of the Lanczos iteration, then

$$
\lambda_{n}^{(n)} \leq \theta_{j}^{(n)} \leq \lambda_{n}^{(n)}+\frac{\left(\lambda_{1}^{(n)}-\lambda_{n}^{(n)}\right) C_{2}}{c_{j-1}\left(1+2 C_{1}\right)^{2}},
$$

where $W_{j}$ is the matrix representation of the projection of $A_{n}$ onto the Krylov subspace $\left[q_{1}, q_{2}, \ldots, q_{j}\right]$ generated by the Lanczos iteration,

$$
C_{1}=\frac{\lambda_{n-1}^{(n)}-\lambda_{n}^{(n)}}{\lambda_{1}^{(n)}-\lambda_{n-1}^{(n)}}, \quad C_{2}=\frac{1-\left|q_{1}^{T} z_{n}^{(n)}\right|^{2}}{\left|q_{1}^{T} z_{n}^{(n)}\right|^{2}},
$$

and $c_{j-1}(x)$ is the Chebyshev polynomial of degree $j-1$.

According to Theorem 3.3, the convergence rate of the estimate of the smallest eigenvalue depends on $C_{1}$. If the constant $C_{1}$ is large, then the Lanczos method will converge very quickly. As a corollary to Theorem 3.2, we can conclude that the Lanczos method, when applied to find the minimum eigenvalue of the preconditioned Toeplitz matrix $Q_{n}^{(k)-1}\left(T_{n}-\rho_{k}^{(n)} I_{n}\right) Q_{n}^{(k)-T}$ with clustered spectra, converges sufficiently fast. The idea of the proof is to select a polynomial that annihilates the $\left(N_{2}-1\right)$ extreme eigenvalues of the preconditioned Toeplitz matrix and the polynomial is large at $\lambda_{n}^{(n)}$ in comparison to its value at the remaining clustered eigenvalues between $1-\epsilon$ and $1+\epsilon$. We have the following corollary.

Corollary 3.4. Let $f$ be a positive continuous function on $[0, \pi]$ and $\left\{T_{n}\right\}$ be a sequence of Toeplitz matrices generated by $f$. If $\inf _{n}\left|\alpha_{n}-\rho_{0}^{(n)}\right| \geq \delta>0$, where $\delta$ is a constant independent of $n$, then for any given $\epsilon>0$ there exist positive integers $N_{1}$ and $N_{2}$ such that for all $n>N_{1}, k \geq 0$ and $j>N_{2}-1$,

$$
\lambda_{n}^{(n)} \leq \theta_{j}^{(n)} \leq \lambda_{n}^{(n)}+\frac{\left(1+\epsilon-\lambda_{n}^{(n)}\right) C_{2}}{c_{j-N_{2}-1}\left(1+2 C_{1}\right)^{2}},
$$

where $\lambda_{n}^{(n)}$ and $z_{n}^{(n)}$ are the smallest eigenvalues and its corresponding orthonormal eigenvector of $Q_{n}^{(k)-1}\left(T_{n}-\rho_{k}^{(n)} I_{n}\right) Q_{n}^{(k)-T}$, respectively, $\theta_{j}^{(n)}$ is the smallest eigenvalue of the matrix $W_{j}$ obtained after $j$ steps of the Lanczos iteration, and

$$
C_{1}=\frac{1-\epsilon-\lambda_{n}^{(n)}}{2 \epsilon}, \quad C_{2}=\frac{\sum_{\ell \text { such that } \lambda_{n}^{(\ell)} \in[1-\epsilon, 1+\epsilon]}\left|q_{1}^{T} z_{n}^{(\ell)}\right|^{2}}{\left|q_{1}^{T} z_{n}^{(n)}\right|^{2}} .
$$

Here the matrix $W_{j}$ is the matrix representation of the projection of $Q_{n}^{(k)-1}\left(T_{n}-\right.$ $\left.\rho_{k}^{(n)} I_{n}\right) Q_{n}^{(k)-T}$ onto the Krylov subspace $\left[q_{1}, q_{2}, \ldots, q_{j}\right]$ generated by the Lanczos iteration.

According to Corollary 3.4, the magnitude $C_{1}$ is sufficiently large if $\epsilon$ is small and $c_{j-N_{2}-1}\left(1+2 C_{1}\right)$ grows exponentially as $j$ increases, therefore the second term of 
the right-hand side inequality (3.3) is sufficiently small for large $j$. We note that the upper bound in (3.3) is tighter than that in (3.2). Thus we expect that the smallest eigenvalue estimate of the preconditioned Toeplitz matrix converges very quickly.

For the outside loop of the PL algorithm, Morgan and Scott [17] showed that the method converges at an asymptotically quadratic rate.

TheOREm 3.5 (Morgan and Scott [17]). Assume that the matrices $P_{n}^{(k)}$ and $P_{n}^{(k)-1}$ in the preconditioned Lanczos algorithm, as stated in Table 1.1, are uniformly bounded in $\ell_{2}$-norm. The iterate $\rho_{k}^{(n)}$ converges to an eigenvalue of $T_{n}$ at an asymptotically quadratic rate.

By combining the results in Lemma 3.1, Corollary 3.4, and Theorems 3.2 and 3.5 , it follows that the inner loop of the PL algorithm will converge very quickly and the iterate will also converge to the minimum eigenvalue of $T_{n}$ at an asymptotically quadratic rate.

We now consider the cost of each Lanczos iteration. For a discussion of Lanczos method, see Golub and Van Loan [8]. It is known that the cost per iteration in the Lanczos method is about $8 n$ operations plus the cost of computing $Q_{n}^{(k)-1}\left(T_{n}-\right.$ $\left.\rho_{k}^{(n)} I_{n}\right) Q_{n}^{(k)-T} v$ for some vectors $v$. Since the matrix $Q_{n}^{(k)}$ is equal to $\Psi_{n}\left(\Delta_{n}-\right.$ $\left.\rho_{k}^{(n)} I_{n}\right)^{1 / 2}$, the matrix-vector multiplications $Q_{n}^{(k)-1} v$ and $Q_{n}^{(k)-T} v$ can be done in $O(n \log n)$ real operations. Since $T_{n}$ and $v$ are both real, the Toeplitz matrix-vector product $T_{n} v$ will also be real. We would like to avoid the added computational work of using complex arithmetic to compute real results. However, we can embed the Toeplitz matrix $T_{n}$ into a larger matrix that can be diagonalized by the sine transform matrix. Thus the matrix-vector product $T_{n} v$ can be computed in $O(n \log n)$ real operations. The details can be found in $[3,4]$. Hence the cost of each Lanczos iteration is of $O(n \log n)$ operations. Moreover, the basic tool of the algorithm is the FST. Since the FST is highly parallelizable, the method can be expected to perform efficiently on a parallel machine for large-scale or real-time applications.

4. An application to signal processing. The input $\left\{x_{k}\right\}$ and the output $\left\{y_{k}\right\}$ of a transversal filter of order $n$ are related by

$$
y_{r}=\sum_{k=0}^{n-1} w_{k} x_{r-k} .
$$

In signal processing problems it is often necessary to estimate the filter coefficients

$$
\left\{w_{0}, w_{1}, \ldots, w_{n-1}\right\}
$$

given observed values $\left\{x_{1}, x_{2}, \ldots, x_{m}\right\}$ and $\left\{y_{1}, y_{2}, \ldots, y_{m}\right\}$ of the input and output, where $m>n$. One way to do this is to choose $\left\{w_{0}, w_{1}, \ldots, w_{n-1}\right\}$ so as to minimize

$$
\sigma\left(w_{0}, w_{1}, \ldots, w_{n-1}\right)=\sum_{r=1}^{m}\left(y_{r}-\sum_{k=0}^{n-1} w_{k} x_{r-k}\right)^{2},
$$

where it is assumed that $x_{j}=0$ if $j \leq 0$. An elementary argument shows that $\left\{w_{i}\right\}_{i=1}^{n-1}$ should be chosen so that

$$
\sum_{j=1}^{n} a_{i j} w_{j-1}=\sum_{r=1}^{m} y_{r} x_{r-i+1}, \quad 1 \leq i \leq n,
$$


where

$$
a_{i j}=\sum_{r=1}^{m} x_{r-i+1} x_{r-j+1} .
$$

The matrix $A_{n}=\left[a_{i j}\right]_{i, j=1}^{n}$ is given by $A_{n}=X^{T} X$, where $X$ is the $m \times n$ Toeplitz matrix

$$
X=\left[\begin{array}{ccccc}
x_{1} & 0 & \cdots & 0 & 0 \\
x_{2} & x_{1} & \ddots & & 0 \\
\vdots & x_{2} & x_{1} & \ddots & \vdots \\
\vdots & \ddots & \ddots & \ddots & 0 \\
x_{n} & \ddots & \ddots & \ddots & x_{1} \\
\vdots & \ddots & \ddots & \ddots & \vdots \\
x_{m-1} & \ddots & \ddots & \ddots & x_{m-n} \\
x_{m} & x_{m-1} & \cdots & x_{m-n+2} & x_{m-n+1}
\end{array}\right]
$$

The matrix $X^{T} X$ is called the normal equations matrix or the information matrix of the corresponding least-squares problem [7,20]. It is an approximation to the correlation matrix of the input signal data. We are interested in computing the eigenvalues of $X^{T} X$ because, for example, the smallest and the largest eigenvalues of $X^{T} X$ are related to the accuracy of the least-squares computations and the stability of least-squares algorithms $[7,20]$. We see that the matrix $X$ has Toeplitz structure. However, the matrix $A_{n}$ is non-Toeplitz in general.

Noting from Lemma 2.3, the minimizer $P_{n}$ of $\left\|S_{n}-A_{n}\right\|_{F}$ over all $S_{n} \in \mathbf{S}$ is still well defined and can be obtained in $O(m \log n)$ operations. Therefore we can use the optimal sine transform-based preconditioner $P_{n}$ to precondition the normal equations matrix $A_{n}$ in the PL algorithm. Using the procedures in [18] and Theorem 3.2, it can be proved that under some practical assumptions in signal processing, the spectra of these preconditioned matrices in the PL algorithm are clustered around 1. Therefore, we expect that the preconditioned Lanczos method will converge very quickly.

5. Numerical results. In this section, we test the performance of the PL algorithm for computing the minimum eigenvalue of a symmetric positive definite Toeplitz matrix. We test the performance on two types of Toeplitz matrices.

(i) The Kac-Murdock-Szegö (KMS) matrix:

$$
\left[T_{n}\right]_{j, k}=t_{j-k}=\eta^{|j-k|}, \quad 0<\eta<1
$$

discussed in [9] and [12]. The eigenvalues of the KMS matrix can be computed quite easily. It was shown in [12] that if

$$
\sin (n+1) \psi-2 \eta \sin n \psi+\eta^{2} \sin (n-1) \psi=0,
$$

then the quantity

$$
\lambda=\frac{1-\eta^{2}}{1-2 \eta \cos \psi+\eta^{2}}
$$


is an eigenvalue of the KMS matrix. It is well known that the generating function for the KMS matrices is given by

$$
f(\theta)=\frac{1-\eta^{2}}{1-2 \eta \cos \theta+\eta^{2}}
$$

see [12]. The generating function is a positive continuous function on $[0, \pi]$.

(ii) The Toeplitz matrix $T_{n}$ :

$$
\left[T_{n}\right]_{j, k}=t_{j-k}= \begin{cases}1+\frac{\pi^{4}}{5}, & j=k, \\ (-1)^{|j-k|}\left(\frac{4 \pi^{2}}{|j-k|^{2}}-\frac{24}{|j-k|^{4}}\right), & j \neq k,\end{cases}
$$

discussed in [4]. The corresponding generating function is given by $f(\theta)=$ $\theta^{4}+1$ for $-\pi \leq \theta \leq \pi$. We have used this Toeplitz matrix as an example to test the performance of preconditioned conjugate gradient method. According to Theorem 1, we note that the minimum eigenvalue of $T_{n}$ is bounded below by 1 .

In the tests, we used the $\hat{j}$ th unit vector as the starting vector $x_{0}$ (cf. Table 1.1) in the PL algorithm, where $\hat{j}$ is the index for which the minimum of $\left\{\left[\Delta_{n}\right]_{j, j}\right\}_{j=1}^{n}$ is attained. The required residual tolerance is $1 \times 10^{-7}$.

Figures 5.1 and 5.2 show the convergence behavior of the PL algorithm and the standard Lanczos algorithm. We plot the relative error between the $\rho_{k}^{(n)}$ and the minimum eigenvalue of the tested Toeplitz matrix against the iteration number. We recall that the main cost per iteration in the Lanczos method is the cost of computing $Q_{n}^{(k)}\left(T_{n}-\rho_{k}^{(n)} I_{n}\right) Q_{n}^{(k)-T} v$ for some vectors $v$. Therefore the iteration number required for convergence means the number of matrix-vector multiplications required in the Lanczos method. The minimum eigenvalue of the tested Toeplitz matrix is computed by the formula in example (i) or by the QR method in example (ii). We see from both Figures 5.1 and 5.2 that the standard Lanczos algorithm converges very slowly. The number of Lanczos iterations required for preconditioned Toeplitz matrices is significantly less than that required for nonpreconditioned Toeplitz matrices. Moreover, the relative error in the standard Lanczos algorithm is larger than that computed in the PL algorithm. Therefore, the sine transform preconditioning can speed up the convergence rate of the Lanczos method.

We compare the optimal sine transform based preconditioner $P_{n}$ with the sine transform based preconditioner $K_{n}$ derived by Boman and Koltracht, as was done in [5] also. From Figures 5.1 and 5.2, the convergence rate of the PL method using $P_{n}$ is the same as that using $K_{n}$, especially when $n$ is large. However, we remark that for an arbitrary Hermitian matrix $A_{n}, K_{n}$ may not satisfy

$$
\lambda_{\min }\left(A_{n}\right) \leq \lambda_{\min }\left(K_{n}\right) \leq \lambda_{\max }\left(K_{n}\right) \leq \lambda_{\max }\left(A_{n}\right) .
$$

Therefore, the starting value and the starting vector of the PL method using $K_{n}$ in these tests are chosen from those of the PL method using the optimal sine transformbased preconditioner $P_{n}$ as $P_{n}$ satisfies (5.1) (cf. Lemma 1). This shows that our sine transform-based preconditioners may be more applicable in the computation of the minimum eigenvalue of a Toeplitz matrix.

For circulant preconditioning, the implementation requires the use of the FFTs and therefore requires complex arithmetic. Since $T_{n}$ is real, we avoid the added computational work of using complex arithmetic to compute real results. Therefore, 

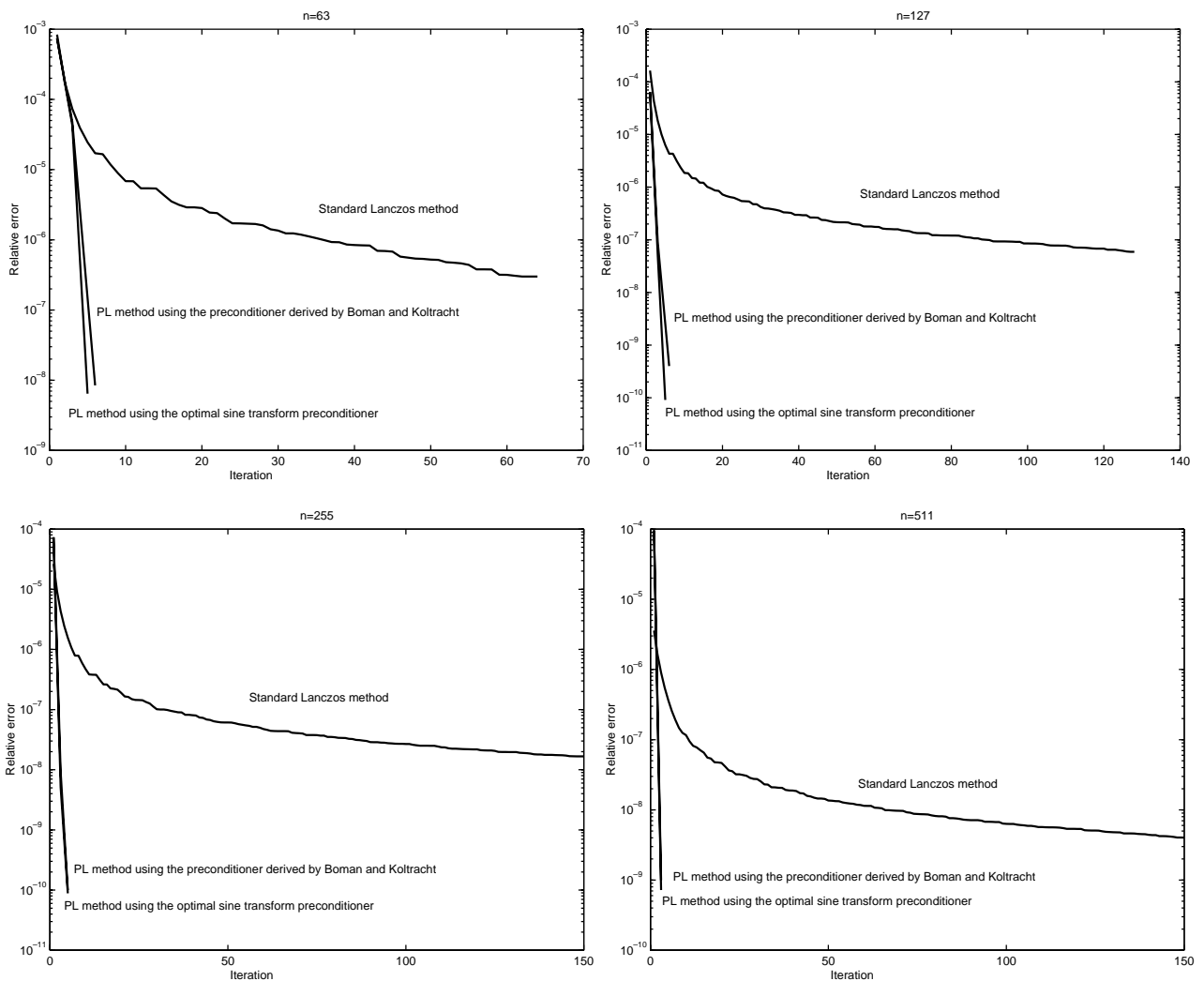

FIG. 5.1. The convergence behavior of $P L$ and standard Lanczos algorithms for Toeplitz matrices (i) $n=63,127,255$, and 511 with $\eta=0.99$.

we do not employ circulant preconditioners in these numerical tests to precondition Toeplitz matrices in the Lanczos iteration.

Next we compare the execution time of the PL method using the optimal sine transform-based preconditioner, the standard Lanczos method, and the method proposed by Trench $[22,23]$ on a Sun Ultra SPARC workstation.

In Figure 5.3, we plot the time required to compute the minimum eigenvalue of Toeplitz matrices in examples (i) and (ii) against the size $n$ of the matrix ( $n=$ $63,127,255,511,1023)$. We use the FST program obtained from the double precision version of FFTPACK in NETLIB to implement the PL method. Since the convergence rate of the PL method using $P_{n}$ is the same as that using the Boman and Koltracht preconditioner $K_{n}$ (cf. Figures 5.1 and 5.2), the time required by using $P_{n}$ or $K_{n}$ to compute the minimum eigenvalue of $T_{n}$ will be almost the same. From Figure 5.3 , we observe that the time required by the PL method is less than those required by the standard Lanczos method and the method proposed by Trench when $n=$ $63,127,255,511,1023$ for examples (i) and (ii). We remark that the time required in each Lanczos iteration depends on the time required to compute FSTs for Toeplitz matrix-vector multiplications. For any $n$-vector $v$, the sine transform matrix-vector product $\Psi_{n} v$ requires $O(n \log n)$ operations [14, p. 155]. In particular, if $n=2^{\nu}-1$ for positive integers $\nu$, then Yip and Rao $[24,25]$ showed that the sine transform matrixvector product can be done more efficiently using the sparse-matrix factorization 

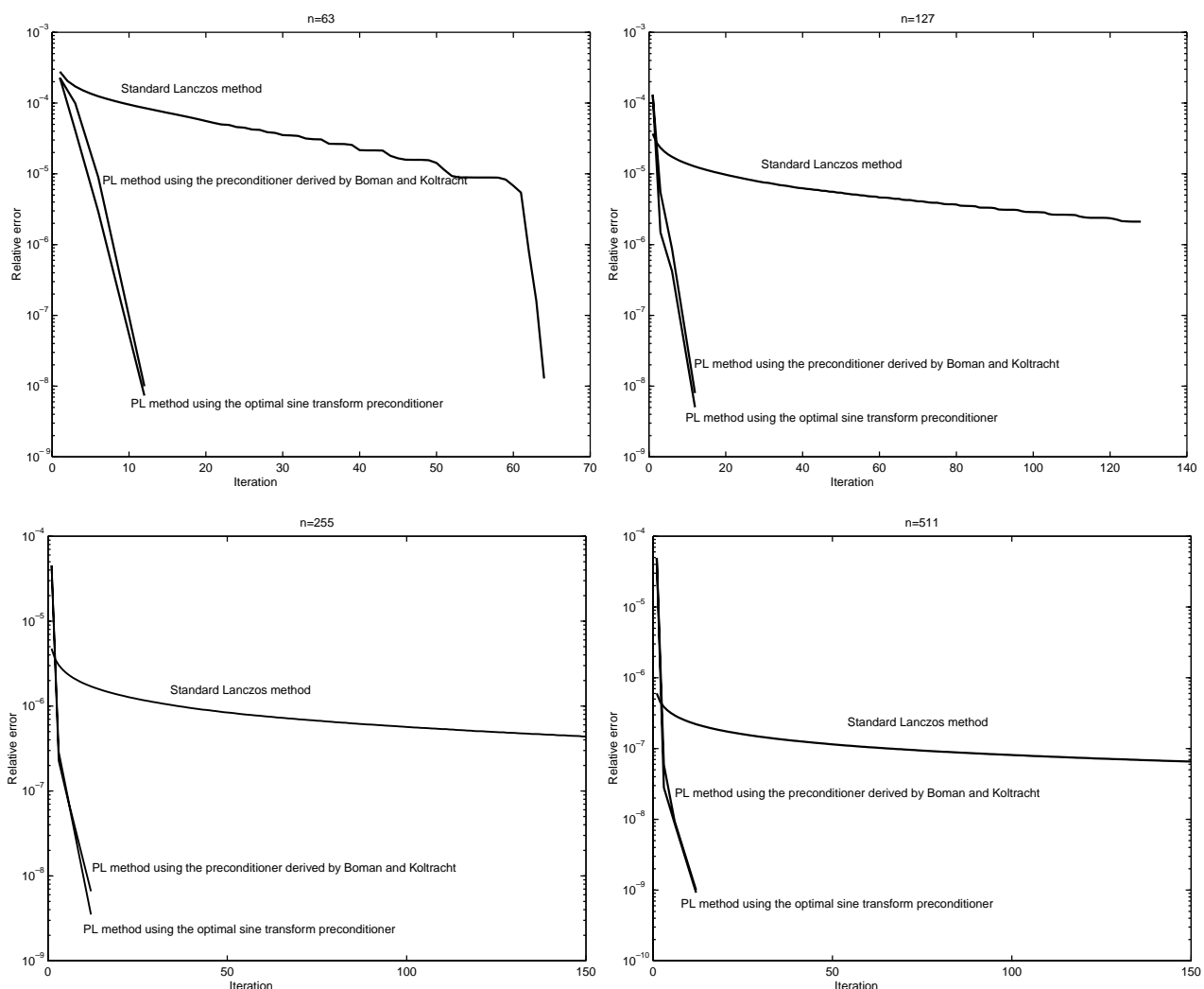

FIG. 5.2. The convergence behavior of PL and standard Lanczos algorithms for Toeplitz matrices (ii) $n=63,127,255$, and 511 .

technique. It requires $(2 n \log n-4 n+4)$ real additions and $((n / 2) \log n-n+1)$ real multiplications, see $[24,25]$. In this case, we note that the cost of each Lanczos iteration is about ( $15 n \log n-19 n+31)$ real operations (additions plus multiplications) [8], whereas the cost of the method proposed by Trench is about $\left(2 n^{2}+n\right)$ real operations [22]. Thus when $n=2^{\nu}-1$, where $\nu$ is large, we expect that the time required by our method will be less than that required by Trench's method.

Finally, we also test our method on matrices of the form $X^{T} X$ where $X$ is as in (4.1), with $m=1024$ and $n=127$. We consider the case with $\left\{x_{1}, \ldots, x_{1024}\right\}$ generated by the second-order autoregressive process

$$
x_{k}-1.4 x_{k-1}+0.5 x_{k-2}=\phi_{k} .
$$

Here $\left\{\phi_{k}\right\}$ is a Gaussian process with mean zero and variance one, and $E\left(\phi_{j} \phi_{k}\right)=$ $\delta_{j k}$. We remark that the sine transform-based preconditioner derived by Boman and Koltracht is not defined in this case since the matrix $X^{T} X$ is not Toeplitz. Figure 5.4 shows the convergence behaviour of the PL algorithm and the standard Lanczos algorithm. Again we observe that the standard Lanczos algorithm requires more iterations and the relative error in the standard Lanczos algorithm is larger than that computed in the PL algorithm.

In summary, these preliminary experiments suggest that the preconditioned Lanczos algorithm with optimal sine transform preconditioning is an efficient and effective 

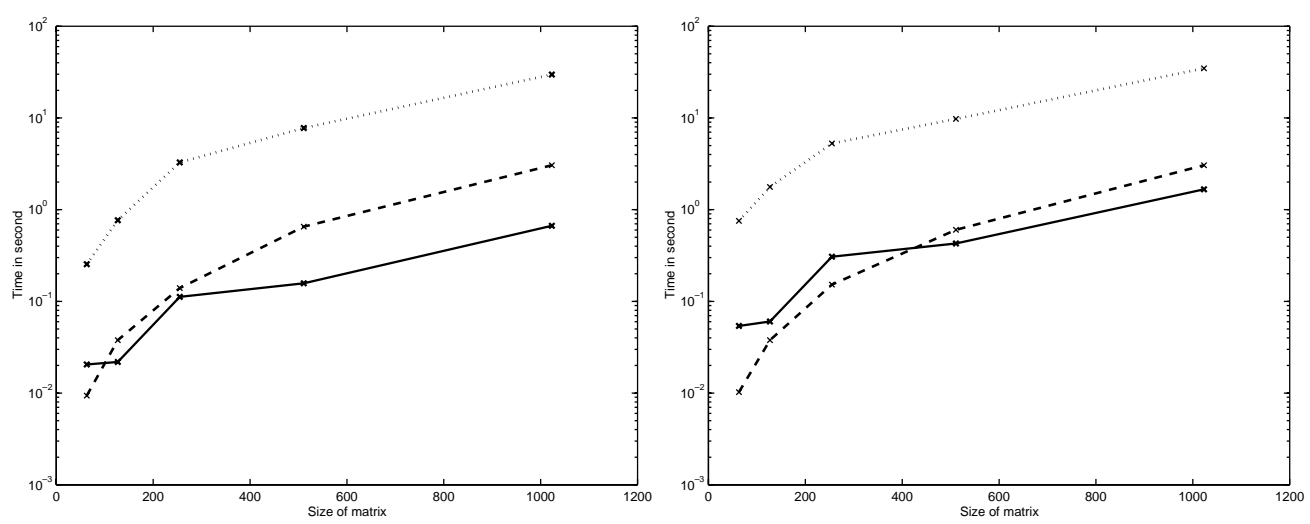

FIG. 5.3. Time (in seconds) required to compute the minimum eigenvalue of the Toeplitz matrix (i) left and (ii) right (..... standard Lanczos method, - - Trench's method, and - - the PL method).

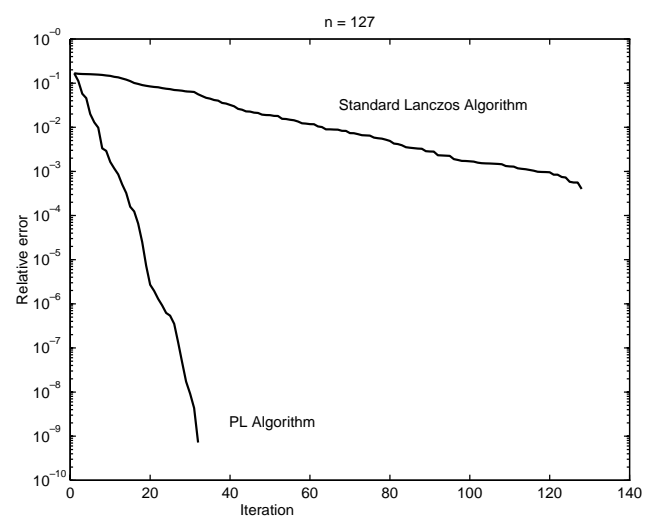

FIG. 5.4. The convergence behavior of $P L$ and standard Lanczos algorithms for $X^{T} X$.

method for computing the minimum eigenvalue of symmetric positive-definite Toeplitz matrices of order $n=2^{\nu}-1$ for positive integers $\nu$.

Acknowledgment. We would like to thank the referees for their valuable comments.

\section{REFERENCES}

[1] G. Ammar And W. GragG, Superfast solution of real positive definite Toeplitz systems, SIAM J. Matrix Anal. Appl., 9 (1988), pp. 61-76.

[2] D. Bini And F. Di Benedetto, A new preconditioner for the parallel solution of positive definite toeplitz systems, in Proceedings Second ACM Symposium on Parallel Algorithms and Architectures, Crete, Greece, 1990, pp. 220-223.

[3] E. Boman and I. Koltracht, Fast transform based preconditioners for Toeplitz equations, SIAM J. Matrix Anal. Appl., 16 (1995), pp. 628-645.

[4] R. Chan And M. NG, Conjugate gradient methods for Toeplitz systems, SIAM Rev., 38 (1996), pp. 427-482.

[5] R. Chan, M. NG, and C. Wong, Sine transform based preconditioners for symmetric Toeplitz systems, Linear Algebra Appl., 232 (1996), pp. 237-259.

[6] G. Cybenko And C. VAN LoAn, Computing the minimum eigenvalue of a symmetric positive definite Toeplitz matrix, SIAM J. Sci. Stat. Comput., 7 (1986), pp. 123-131.

[7] W. Ferng, G. Golub, And R. Plemmons, Adaptive Lanczos methods for recursive condition 
estimation, Numer. Algorithms, 1 (1991), pp. 1-19.

[8] G. Golub and C. Van Loan, Matrix Computations, 2nd ed., The Johns Hopkins University Press, Baltimore, MD, 1989.

[9] U. Grenander and G. Szegö, Toeplitz Forms and Their Applications, 2nd ed., Chelsea Publishing, New York, 1984.

[10] Y. Hu And S. Kung, Toeplitz eigensystem solver, IEEE Trans. Acoustics Speech Signal Process., ASSP-33 (1985), pp. 1264-1271.

[11] T. HuCKLE, Fast transforms for tridiagonal linear equations, BIT, 34 (1994), pp. 99-112.

[12] M. Kac, W. Murdock, And G. Szegö, On the eigenvalues of certain Hermitian forms, J. Rational Mech. Anal., 2 (1953), pp. 767-800.

[13] T. Kailath And A. SaYed, Displacement structure: Theory and applications, SIAM Rev., 37 (1995), pp. 297-386.

[14] A. Jain, Fundamentals of Digital Image Processing, Prentice-Hall, Englewood Cliffs, NJ, 1989.

[15] N. LeVINSON, The Wiener RMS (root mean square) error criterion in filter design and prediction, J. Math. Phys., 25 (1946), pp. 261-278.

[16] W. Mackens And H. Voss, The minimum eigenvalue of a symmetric positive-definite Toeplitz matrix and rational Hermitian interpolation, SIAM J. Matrix Anal. Appl., 18 (1997), pp. $521-534$.

[17] R. Morgan and D. Scott, Preconditioning the Lanczos algorithm for sparse symmetric eigenvalue problems, SIAM J. Sci. Comput., 14 (1993), pp. 585-593.

[18] M. NG, Fast iterative methods for solving Toeplitz-plus-Hankel least squares problems, Electron Trans. Numer. Anal., 2 (1994), pp. 154-170.

[19] B. Parlett, The Symmetric Eigenvalue Problem, Prentice-Hall, Englewood Cliffs, NJ, 1980.

[20] D. Pierce and R. Plemmons, Tracking the condition number for RLS in signal processing, Math. Control Signals Systems, 5 (1992), pp. 23-39.

[21] Y. SAAD, Numerical Methods for Large Eigenvalue Problems, Manchester University Press, New York, 1992.

[22] W. Trench, Numerical solution of the eigenvalue problem for Hermitian Toeplitz matrices, SIAM J. Matrix Anal. Appl., 9 (1988), pp. 291-303.

[23] W. TREnch, Numerical solution of the eigenvalue problem for efficiently structured Hermitian matrices, Linear Algebra Appl., 154-156 (1991), pp. 415-432.

[24] P. YIP AND K. RAO, A fast computational algorithm for the discrete sine transform, IEEE Commun. Trans., 28 (1980), pp. 304-307.

[25] P. YIP AND K. RAO, Fast decimation-in-time algorithms for a family of discrete sine and cosine transforms, Circuits Systems Signal Process., 3 (1984), pp. 387-408. 